\title{
SIGNIFICADO Y TRATAMIENTO DEL PAISAJE EN LAS POLITICAS DE PROTECCIÓN DE ESPACIOS NATURALES EN ESPAÑA
}

\author{
Alfonso Mulero Mendigorri \\ Departamento de Geografía y Ciencias del Territorio. Universidad de Córdoba \\ alfonso.mulero@uco.es
}

\section{RESUMEN}

El objetivo esencial de este trabajo consiste en analizar la consideración del paisaje en las políticas españolas de protección de espacios naturales. Con tal intención, se efectúa un recorrido sintético a través de las sucesivas normas estatales que se han ocupado de la cuestión, para, a continuación, proceder a valorar la implantación territorial del Paisaje Protegido, la categoría protectora de referencia en las más recientes leyes de conservación del medio natural.

Palabras clave: Paisaje, paisaje protegido, espacios naturales protegidos, patrimonio natural.

\section{ABSTRACT}

The main objective of this paper is to analyze the consideration of the landscape in Spanish natural protected areas policies. With this intention, it makes a synthetic route through successive state rules that have addressed the matter to then proceed to evaluate the implementation of the Protected Landscape, category included in the latest environmental conservation laws.

Key words: Landscape, protected landscape, protected natural areas, natural heritage.

Fecha de recepción: septiembre 2011.

Fecha de aceptación: enero 2013. 


\section{INTRODUCCIÓN Y OBJETIVOS}

En la actualidad asistimos a un inusitado interés científico y técnico por el estudio del paisaje, así como por su protección y puesta en valor. En paralelo, la eclosión de estudios y de iniciativas políticas ha traído aparejada una cierta confusión conceptual, debida en parte a los distintos «lenguajes» que emplean las numerosas disciplinas interesadas en el tema. Sin embargo, también contribuye a esta falta de entendimiento la diversidad de instancias y órganos administrativos con competencias en ordenación y gestión del paisaje. Y todo ello sin que el debate interdisciplinar o la coordinación interadministrativa estén presentes en la medida necesaria.

En este contexto, son muchas las cuestiones necesitadas de clarificación y reflexión. A nuestro juicio, una de notable importancia es la relación existente entre la valoración del paisaje y la declaración de espacios naturales protegidos, presidida siempre por una considerable ambigüedad. No debe olvidarse que el interés por la preservación de paisajes excepcionales ha estado presente en las políticas españolas de espacios naturales protegidos desde el propio origen de las mismas, y que en las últimas décadas, por diversas razones que se indicarán, se ha convertido en asunto de primer orden en las grandes iniciativas protectoras emprendidas en el Estado español.

Desde esta perspectiva, en el presente trabajo se abordan dos grandes cuestiones; de un lado -y en un recorrido necesariamente sintético- nos ocupamos del tratamiento otorgado al paisaje en las normas básicas reguladoras de la protección de los espacios naturales. A continuación -y ya en el terreno de los logros concretos- nos detendremos en el análisis de otro asunto esencial, cual es la aplicación de la figura de Paisaje Protegido en España; una figura cuya inclusión en la Ley de Conservación de Espacios Naturales de 1989 supuso un hito de primer orden, sobre todo por lo que tuvo de reconocimiento oficial explícito de la necesidad de identificar y proteger determinados paisajes concretos. Con tal fin, hemos estudiado la implantación de esta categoría protectora en el territorio español, los criterios empleados en la selección y gestión de los ámbitos catalogados como paisajes protegidos, e, igualmente, su significado en el contexto del vasto inventario estatal de áreas protegidas.

Es preciso aclarar que, debido al objetivo específico de este trabajo, el análisis territorial se ha centrado expresamente en la figura de Paisaje Protegido, sin que ello implique obviar que existen otras categorías protectoras -muy destacadamente los Parques Naturales- cuya profusa aplicación ha conllevado una notable protección de la riqueza paisajística presente en extensos territorios, bien es cierto que de forma indirecta -es decir, sin una pretensión explícita-, y a través, por ejemplo, de la aplicación de las normas reguladoras de usos y actividades contenidas en los Planes de Ordenación de los Recursos Naturales (PORN).

Finalmente, conviene recordar que las importantes aportaciones recogidas en el Convenio Europeo del Paisaje (2000) en materia de definición, análisis y gestión del paisaje, no han tenido traslación a la legislación española de áreas protegidas hasta 2007, con la promulgación de la Ley del Patrimonio Natural y la Biodiversidad. Para entonces ya se había producido la declaración de la gran mayoría de los actuales espacios protegidos, y éstos han venido siendo gestionados en función de la anterior legislación, tanto estatal como autonómica, por lo general bastante pobre en materia de regulación y protección paisajística. En consecuen- 
cia, es de esperar que los efectos concretos de las disposiciones del Convenio Europeo -que se detallan en páginas posteriores- no se dejen sentir de forma explícita en el sistema español de áreas protegidas hasta los próximos años.

\section{LA EVOLUCIÓN DEL MARCO NORMATIVO Y LA CONCEPCIÓN DEL PAISAJE}

\section{Los antecedentes preautonómicos}

En el contexto general de las políticas de espacios naturales protegidos aplicadas en España hasta la fecha es preciso distinguir dos etapas bien diferenciadas. La primera se extiende desde la promulgación de la Ley de Creación de Parques Nacionales (1916) -considerada el punto de partida de la legislación específica sobre el particular- hasta el diseño del Estado de las Autonomías apoyado en la Constitución Española de 1978; desde esta última fecha se inicia una nueva andadura marcada por el traspaso progresivo de las competencias en materia de conservación de la naturaleza desde el Estado a las Comunidades Autónomas, bajo cuya iniciativa -muy dispar como es sabido- se ha producido la regulación, declaración y gestión del complejo sistema actual de espacios naturales protegidos (Mulero, 2002). Un sistema que se concreta en la existencia de aproximadamente 1600 declaraciones con una extensión conjunta que sobrepasa los 6,2 millones de hectáreas, sin considerar las áreas protegidas por los diversos acuerdos internacionales.

A los efectos que ahora nos ocupan es importante recordar que la salvaguarda de determinados valores y cualidades paisajísticas fue un factor esencial ya desde las primeras iniciativas de declaración de espacios protegidos, conforme a lo dispuesto en la pionera ley y en sus instrumentos de desarrollo (Solé y Bretón, 1986; Gómez, 1999; Casado, 2000; Mata, 2000; Lasagabaster y Lazcano, 2004). En tal sentido, sendos trabajos recientes de García (2009) y de Ortega y García (2009) se han ocupado del significado que determinados valores simbólicos e identitarios vinculados al paisaje tuvieron en la creación de los primeros espacios protegidos y, destacadamente, en el pionero Parque Nacional de la Montaña de Covadonga (1918); recuérdese que sus atributos paisajísticos y su belleza estética fueron ensalzados reiteradamente por Pedro Pidal, Marqués de Villaviciosa de Asturias y principal impulsor de la declaración de este paraje.

No obstante, durante la primera etapa de la política española de espacios protegidos, la vinculación entre el paisaje y los espacios naturales que se iban declarando quedó limitada al reconocimiento de la importancia de aquél, de forma más o menos genérica, y a la necesidad de su salvaguarda en virtud de consideraciones de muy variada índole. En ningún momento se estipularon directrices específicas orientadas a la defensa del paisaje ni figuras concretas de protección paisajística.

Por el contrario, se inició un camino de confusión entre ambos conceptos -paisaje y espacio natural protegido- que incluso llevó a la propia administración forestal a considerarlos como realidades equivalentes. Un ejemplo paradigmático de tal dislate lo encontramos en el denominado Inventario Nacional de Paisajes Sobresalientes, elaborado por el Instituto Nacional de Conservación de la Naturaleza (ICONA, 1977) con la intención de recopilar en un catálogo general aquellos espacios naturales españoles merecedores de protección, siguiendo los criterios imperantes en la fecha. 
En la misma línea, la Ley de Espacios Naturales Protegidos (1975) -el segundo gran texto normativo de referencia en la etapa preautonómica- obvió casi por completo las referencias al valor del paisaje y a la necesidad de su protección y gestión. De hecho, la principal alusión al mismo se encuentra en la definición de la figura de Parque Nacional (art. $3^{\circ}$ ), donde se recoge que: «Son Parques Nacionales los espacios naturales de relativa extensión que se declaren por ley como tales por la existencia en los mismos de ecosistemas primigenios que no hayan sido sustancialmente alterados por la penetración, explotación y ocupación humana, y donde las especies animales y vegetales, así como los lugares y las formaciones geomorfológicas, tengan un destacado interés cultural, educativo o recreativo, $o$ en los que existan paisajes naturales de gran belleza» (la cursiva es nuestra).

\section{La etapa autonómica y el desarrollo de los primeros instrumentos orientados a la protec- ción del paisaje}

Como se ha dicho, tras la promulgación de la Constitución de 1978 se inició el proceso de traspaso de competencias en materia de conservación de la naturaleza a las Comunidades Autónomas. La transición entre los sistemas de espacios protegidos centralizado y autonómico se caracterizó por la temprana aparición de diversas leyes regionales de protección, impulsadas por cinco Comunidades Autónomas ${ }^{1}$, que se anticiparon de este modo a la aprobación de la ley marco que vendría a regular la nueva situación político administrativa del Estado (Ley 4/1989, de 27 de marzo, de Conservación de Espacios Naturales y de la Flora y Fauna Silvestres). En general, las iniciativas citadas incurrieron en la precipitación y en una clara falta de previsión de los acontecimientos que iban a producirse en un futuro inmediato (Mulero, 2002), ya que la Constitución atribuye al Estado la facultad de establecer la legislación básica en la materia y, en buena lógica, convenía conocer cuáles iban a ser las estipulaciones del texto que vendría a sustituir a la Ley de 1975, antes de emprender caminos propios. El resultado de tal proceder apresurado fue que las primeras leyes autonómicas fueron derogadas y sustituidas por otras más completas a los pocos años de su aprobación, con la excepción de la catalana que continúa vigente.

Con relación al tratamiento del paisaje, los textos regionales pioneros no introdujeron figuras específicas para su protección, ni tampoco instrumentos o directrices orientadas a un manejo ordenado de los valores paisajísticos de los numerosos espacios naturales protegidos declarados inicialmente. No obstante, la ley catalana recoge cierta predisposición y sensibilidad hacia el tema, según se deprende de algunas disposiciones de su articulado; por ejemplo, en el preámbulo se indica que una de las razones de la necesidad de promulgar la norma autonómica reside en la extraordinaria variedad del medio natural de Cataluña, donde se da cita «(...) una gran parte de las estructuras de paisaje que se encuentran en Europa y en el norte de África (...)»; y, asimismo, se manifiesta la preocupación por los «efectos visuales y estéticos de la degradación y destrucción del paisaje (...)». En el mismo sentido, pero con mayor alcance, el art. $5^{\circ}$ contiene la única disposición realmente operativa, aunque con base en la normativa urbanística, cuando estipula que «La Administración de la Generalidad (...)

1 Estas fueron Cataluña, Canarias, Comunidad Valenciana, Islas Baleares y Navarra. 
podrá formular y tramitar planes especiales para la protección del medio natural y del paisaje, de acuerdo con lo establecido en la legislación urbanística».

En relación con lo dicho, conviene tener presente que los primeros instrumentos y figuras de protección paisajística con incidencia sobre el medio físico-natural se apoyaron antes en la legislación urbanística que en la medioambiental, siendo un buen ejemplo de ello los Planes Especiales de Protección del Medio Físico, aprobados en Andalucía a mediados de los años ochenta. Baste recordar ahora que los planes andaluces, diseñados a escala provincial, supusieron un importante refuerzo protector para el conjunto del suelo no urbanizable, tanto por su corpus de normas de usos y actividades permitidas, como por el establecimiento de los primeros Catálogos Provinciales de Bienes y Espacios Protegidos ${ }^{2}$. Estos se agruparon en nueve categorías, de entre las cuales dos -Paisajes Sobresalientes y Paisajes Agrícolas Singulares-estuvieron orientadas claramente a la salvaguarda de determinados ámbitos en función de valores paisajísticos excepcionales o muy representativos. Los Paisajes Sobresalientes, fueron definidos como «aquellos espacios que se caracterizan por su reconocida singularidad paisajística, frecuentemente apoyada en rasgos geomorfológicos notables. Suelen presentar, asimismo, importantes valores faunísticos y/o botánicos. En general son unidades de relativa uniformidad, que se comportan como emisores o receptores visuales de gran interés científico, cultural o estético». Por su parte, los Paisajes Agrícolas Singulares se concibieron como «áreas representativas, normalmente dehesas, vegas, regadíos, de paisajes agrarios de gran calidad paisajística y/o productiva. Estos espacios se consideran particularmente importantes como articuladores de la actividad agraria circundante».

En definitiva, en el conjunto del territorio andaluz fueron catalogados 36 Paisajes Sobresalientes y 64 Paisajes Agrícolas Singulares, bien es cierto que con criterios dispares en las diferentes provincias como consecuencia de una clara falta de coordinación en la elaboración de los respectivos planes; además, conviene precisar también que si la propia designación de tales espacios -con su correspondiente normativa de usos prohibidos y permitidos- supuso un logro de enorme trascendencia en un momento de escasa protección del medio natural andaluz, el mismo se vio lastrado por la inexistencia de instrumentos para la ordenación y gestión futura de los mismos.

\section{El primer avance sustancial en la protección del paisaje: la Ley de Conservación de los Espacios Naturales de $1989^{3}$}

Puede afirmarse, en suma, que hasta la promulgación de la citada ley en 1989 no comienza a fraguarse una relación estrecha entre la protección del medio natural y la salvaguarda del paisaje. Un análisis pormenorizado del citado texto y de sus instrumentos de desarrollo permite distinguir entre las medidas que pueden tener una incidencia «indirecta» sobre la ordenación y estabilidad paisajística general, de aquellas otras diseñadas para intervenir «directamente» en la conservación de paisajes específicos. Con relación a las primeras han de destacarse las dos que siguen:

2 Los espacios catalogados fueron la base sobre la que se establecería en 1989 el Inventario de Espacios Naturales Protegidos de Andalucia (Ley 2/1989, de 18 de julio, por la que se crea el inventario de espacios naturales protegidos de Andalucía y se establecen medidas adicionales para su protección.

3 Ley 4/1989, de 27 de marzo, de conservación de espacios naturales y de la flora y fauna silvestres. 
$1^{\mathrm{a}} \quad$ La inclusión de la preservación del paisaje como uno de los cuatro principios básicos inspiradores de la ley; a saber: el mantenimiento de los procesos ecológicos esenciales y de los sistemas vitales básicos; la preservación de la diversidad genética; la utilización ordenada de los recursos, garantizando el aprovechamiento sostenido de las especies y de los ecosistemas, su restauración y mejora, y, finalmente, la preservación de la variedad, singularidad y belleza de los ecosistemas naturales y del paisaje.

$2^{\mathrm{a}} \quad$ El establecimiento del Plan de Ordenación de los Recursos Naturales (en adelante PORN), concebido como el instrumento básico para la planificación del medio natural español, con un radio de acción que puede sobrepasar el ámbito estricto de los espacios naturales protegidos. Indirectamente, los PORN pueden desempeñar un importante papel en la ordenación y protección paisajísticas por varias razones: de entrada, gozan de una extraordinaria fortaleza jurídica, pues sus disposiciones prevalecen sobre las de cualquier otro instrumento de planificación territorial o urbanística, y, de otro lado, al ser obligatorios para todos los Parques y Reservas, su incidencia está garantizada, ya que éstas son las categorías dominantes en términos de superficie protegida (Tabla 1)-. En consecuencia, en sí mismos son un excelente instrumento de cara a la identificación y valoración del patrimonio paisajístico existente en los espacios protegidos, o en cualquier ámbito territorial donde se apliquen (Bermejo, 2007). Pero, además, al zonificar el territorio y establecer normas reguladoras de los usos permitidos o prohibidos en cada zona, tanto los PORN como los PRUG condicionan claramente la dinámica futura del paisaje. Se trata de un asunto crucial para entender las transformaciones paisajísticas que se están produciendo en determinados espacios protegidos, para cuya interpretación han de tenerse en cuenta tanto los diferentes criterios empleados en la elaboración de los PORN -en una misma Comunidad Autónoma e incluso entre parques y reservas limítrofes-, como los cambios recientes que, por distintas razones, se vienen introduciendo en las zonificaciones establecidas originalmente; el sistema andaluz de Parques Naturales es buen ejemplo de lo dicho, y algunos casos particulares han sido estudiados en trabajos recientes de Arias (2007), Arias y Garzón (2009), y Mulero (2009), entre otros.

Finalmente, en el contexto que nos ocupa, no se olvide que algunos PORN y PRUG han venido incorporando caracterizaciones sintéticas de los tipos de paisaje presentes en el espacio protegido, así como normas y directrices para la gestión paisajística.

En lo concerniente a la intervención «directa» sobre la protección del paisaje, no cabe duda de que la inclusión de la figura de Paisaje Protegido entre el cuarteto de categorías protectoras de referencia a escala estatal (Parque, Reserva Natural, Monumento Natural y Paisaje Protegido) puede considerarse la principal medida de la ley de 1989. Sin duda, su incorporación supuso, en principio, un salto jurídico de calado, por cuanto vino a reconocer de forma explícita que es necesario proteger determinados paisajes, y que ello debe hacerse en el marco de la legislación medioambiental. Téngase en cuenta, además, que entre las citadas figuras ésta fue la única verdaderamente novedosa, al carecer de antecedentes en la regulación española sobre la materia, pero a la vez se ha convertido en la más polémica y en la que ha tenido un menor desarrollo, en buena medida a causa de la ambigua definición con 
que se la dotó: «Aquellos lugares concretos del medio natural que, por sus valores estéticos y culturales, sean merecedores de una protección especial».

Pese a lo dicho, los Paisajes Protegidos se han ido extendiendo progresivamente y la mayoría de las Comunidades Autónomas cuentan ya con alguno en su territorio, tal y como tendremos ocasión de tratar en páginas posteriores.

\section{La Ley del Patrimonio Natural y de la Biodiversidad ${ }^{4}$ : un importante salto cualitativo auspiciado por el Convenio Europeo del Paisaje}

Es sabido que el Convenio Europeo del Paisaje, firmado en Florencia en 2000, ha deparado hasta la fecha algunas consecuencias importantes en materia de ordenación, regulación y protección paisajística (Zoido, 2010), entre la cuales sobresalen la promulgación de diversas leyes autonómicas específicas (Valencia, Cataluña y Galicia) ${ }^{5}$ y la introducción de disposiciones de interés en algunas normas estatales de notable alcance ${ }^{6}$. Entre éstas, y a los efectos que ahora nos ocupan, hemos de centrarnos en la Ley del Patrimonio Natural y de la Biodiversidad (2007), texto regulador que vino a sustituir al anteriormente tratado; sin duda, la promulgación del Convenio de Florencia y la firme decisión de ratificarlo ${ }^{7}$-con el compromiso que ello implicaría para el Estado español- fue una circunstancia decisiva a la hora de otorgar al paisaje un mayor protagonismo en la renovada estrategia española de conservación de la naturaleza.

El nuevo texto, considerablemente más prolijo que su antecesor, incorpora las contribuciones básicas de éste en la materia y aporta nuevos aspectos esenciales para el conocimiento y la regulación del paisaje. Así, la protección del paisaje sigue reconociéndose como uno de los principios inspiradores de la ley, a la vez que se mantiene el Paisaje Protegido entre las figuras protectoras de referencia, aunque ahora se la dota de una nueva definición en sintonía con lo establecido por la Convención de Florencia: «Paisajes Protegidos son partes del territorio que las Administraciones competentes, a través del planeamiento aplicable, por sus valores naturales, estéticos y culturales, y de acuerdo con el Convenio del paisaje del Consejo de Europa, consideren merecedores de una protección especial» (art. 34). A la vez, se establecen varios objetivos que han de orientar su gestión allí donde se instauren: la conservación de los valores singulares que los caracterizan, la preservación de la interacción armoniosa entre la naturaleza y la cultura en una zona determinada, y, en relación con lo anterior, el mantenimiento de las prácticas de carácter tradicional que contribuyan a la preservación de sus valores y recursos naturales. Por otra parte, recuérdese que el documento del Consejo de Europa insiste en la necesidad de profundizar en la catalogación y conocimiento de los tipos de paisajes identificados. Y en este contexto ha de entenderse la nueva estipulación legal que obliga a que los Planes de Ordenación de los Recursos Naturales incorporen «el

4 Ley 42/2007, de 13 de diciembre.

5 Ley 4/2004, de 30 de junio, de la Generalitat Valenciana, de ordenación del territorio y protección del paisaje; Ley 8/2005, de 8 de junio, de la Generalitat de Cataluña, de protección, gestión y ordenación del paisaje; Ley $7 / 2008$, de 7 de julio, de protección del paisaje de Galicia.

6 Entre ellas la citada Ley 42/2007, del Patrimonio Natural y de la Biodiversidad; la Ley 45/2007, de Desarrollo Sostenible del Medio Rural; el Real Decreto 8/2008 refundido sobre Legislación del Suelo.

7 La ratificación de España se produjo mediante instrumento específico de 30 de noviembre de 2007. 
inventario y definición del estado de conservación de los componentes del patrimonio natural y la biodiversidad, de los ecosistemas y los paisajes en el ámbito territorial de que se trate, formulando un diagnóstico del mismo y una previsión de su evolución futura» (art. 19).

Del mismo modo, la ley del Patrimonio Natural asume algunos principios básicos de la Directiva de Hábitats ${ }^{8}$, entre los que nos interesa destacar el de la utilización del paisaje como instrumento potencial para dotar de coherencia y conectividad a la Red Natura. La traslación a la norma española se concreta en lo siguiente: «(...) se fomentará la conservación de corredores ecológicos y la gestión de aquellos elementos del paisaje o áreas territoriales que resulten esenciales o revistan primordial importancia para la migración, la distribución geográfica y el intercambio genético entre poblaciones de especies de fauna y flora silvestres» (art. 46). Cabe recordar que diversos paisajes protegidos declarados en España han sido concebidos a modo de corredores ecológicos, como tendremos ocasión de tratar en páginas posteriores.

Por último, otra novedad destacable de la ley tiene que ver con el establecimiento de incentivos para las actividades que contribuyan a la protección del paisaje tanto en los espacios naturales protegidos como en otros ámbitos sujetos a acuerdos de custodia del territorio, ya que las mismas pasan a tener la consideración de «externalidades positivas» o servicios derivados de un adecuado manejo de tales territorios. En sentido opuesto, en el repertorio de infracciones tipificadas por la ley se recoge como infracción grave «la instalación de carteles de publicidad o la producción de impactos paisajísticos sensibles en los espacios naturales protegidos» (art. 76, h).

\section{LA FIGURA DE PAISAJE PROTEGIDO: SIGNIFICADO TERRITORIAL, CRITERIOS DE SELEC- CIÓN E INSTRUMENTOS DE GESTIÓN}

Desde que en 1989 se incorporó la figura de Paisaje Protegido al ordenamiento jurídico español, su implantación ha sido lenta aunque progresiva. Las cuatro primeras declaraciones se efectuaron en Murcia en 1990 (Cuatro Calas; Espacios Abiertos e Islas del Mar Menor; Humedal de Ajauque y Rambla Salada; Sierra de las Moreras) y dos décadas más tarde una decena de Comunidades Autónomas han hecho uso de la misma. El balance estatal para el año 2010 cifra en 53 los Paisajes Protegidos existentes, con una extensión global de 150.691 ha (equivalentes a un $2,3 \%$ de la superficie española protegida). No obstante, de cara a una correcta interpretación del actual significado territorial de esta figura protectora conviene tener presentes algunas consideraciones básicas (Figura 1):

- Sin duda, las dificultades conceptuales que envuelven al Paisaje Protegido en España explican, al menos parcialmente, el escaso número de declaraciones producidas en comparación con el resto de categorías protectoras de ámbito estatal (Tabla 1).

- A escala autonómica Canarias, con 27 Paisajes Protegidos, ostenta un claro protagonismo en número de declaraciones y en superficie protegida (38.998, 20 ha), seguida por la Comunidad Valenciana (8 Paisajes Protegidos con 37.439,74 ha). Entre ambas concentran el $66 \%$ de los paisajes declarados hasta el momento, y el $50 \%$ de la exten-

8 Directiva 92/43/CE, de 21 de mayo, relativa a la conservación de los hábitats naturales y de la flora y fauna silvestres 
sión global de los mismos, lo que significa que sin su contribución el catálogo español de Paisajes Protegidos sería francamente pobre (Tabla 2).

- Con carácter general, la figura de Paisaje Protegido ha sido aplicada en ámbitos de tamaño pequeño y mediano, con predominio del intervalo comprendido entre las 100 y las 5.000 hectáreas. Sin embargo, existen notorias excepciones a esta tónica general, como los casos de la Chorrera de Horcajo (37 ha) o del Río Tinto (16.957 ha).

Tabla 1

ESPACIOS NATURALES PROTEGIDOS EN ESPAÑA (2010)

\begin{tabular}{|l|c|c|}
\hline \multicolumn{1}{|c|}{ Figura de protección } & Número de espacios & Superficie (has.) \\
\hline Parques (Nacionales/Naturales) & 171 & $4.055 .040,37$ \\
\hline Reservas Naturales & 280 & $169.501,81$ \\
\hline Monumento Natural & 344 & $98.940,95$ \\
\hline Paisaje Protegido (1) & 53 & $150.691,14$ \\
\hline Otras figuras autonómicas & 835 & 2.470 .495 \\
\hline
\end{tabular}

Fuente: Elaboración propia a partir de datos de Europarc, Ministerio de Medio Ambiente Rural y Marino y Comunidades Autónomas.

(1) No se han contabilizado algunos paisajes protegidos aún no declarados pero en proceso de tramitación.

En consecuencia, en términos cuantitativos es evidente la debilidad de esta categoría protectora, como lo es también en materia de representatividad territorial. Su escasa aplicación en la mayor parte de las Comunidades Autónomas e incluso el nulo uso que han hecho de ella algunas tan significadas como Cataluña, Castilla y León o Madrid, son buena prueba de ello. Pero, además, existe otro factor que le resta credibilidad: la disparidad manifiesta de los criterios y argumentos manejados por los entes autonómicos a la hora de seleccionar las 53 piezas declaradas hasta la fecha. En tal sentido pueden distinguirse, sintéticamente, tres orientaciones diferenciadas:

a) Por una parte, el Paisaje Protegido es entendido como un lugar representativo de la relación histórica entre el hombre y la naturaleza -síntesis de valores naturales, estéticos y culturales-. Esta es la concepción que mejor se ajusta a las definiciones recogidas en las leyes marco de 1989 y 2007, en las que se equipara el valor de los componentes estéticos, culturales y naturales de un territorio; téngase presente que, a diferencia de lo pretendido con las restantes figuras de ámbito estatal, la salvaguarda del medio natural no es el objetivo fundamental del Paisaje Protegido.

Con evidente coherencia, ésta ha sido la perspectiva elegida por las autonomías con mayor número de declaraciones, y un excelente ejemplo de ello lo encontramos en la red canaria, donde extraordinarios paisajes rurales como La Geria (Lanzarote), Tafira (Gran Canaria), Ventejís (El Hierro), Tamanca (La Palma), Orone (La Gomera), etc., exponen de manera singular el equilibrio entre el medio natural y las actividades agrarias isleñas. En la misma línea, los Paisajes Protegidos de la Comunidad Valenciana son, en términos generales, ámbitos caracterizados por una prolongada presencia humana donde, pese a todo, ha pervivido una relación armónica entre su patrimonio 


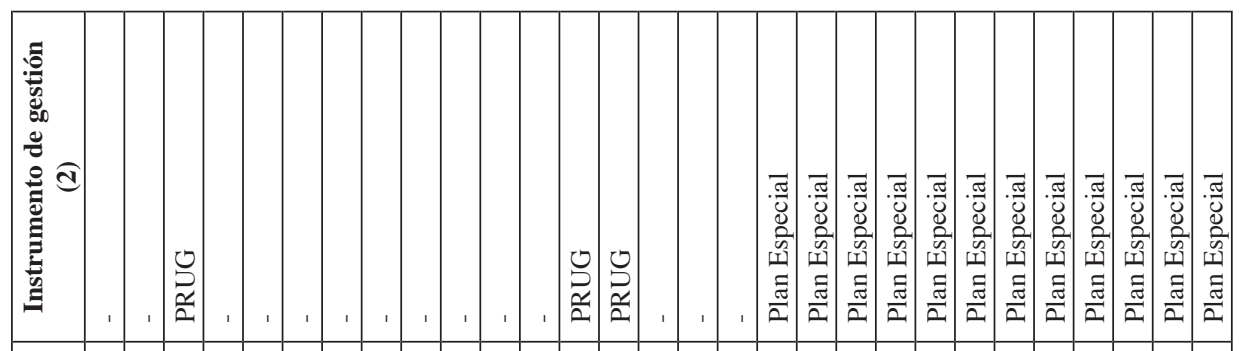

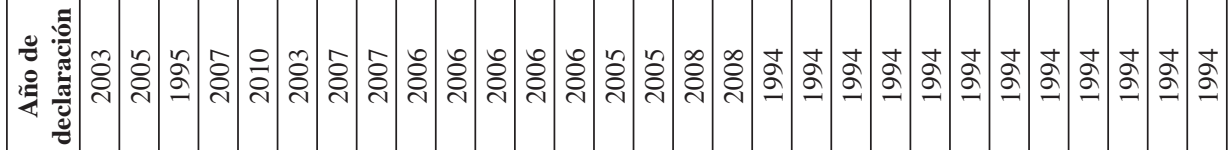
율

i

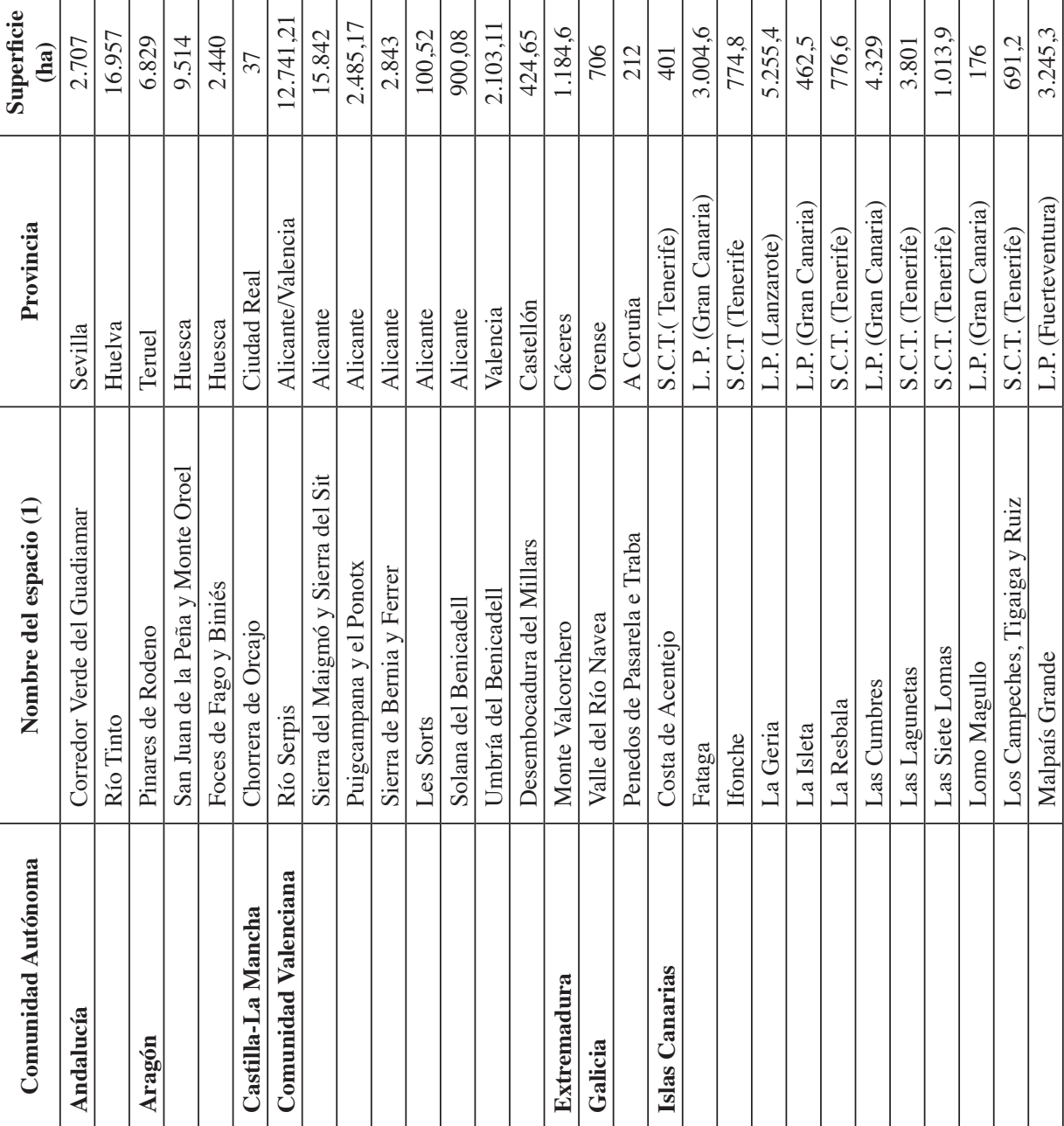




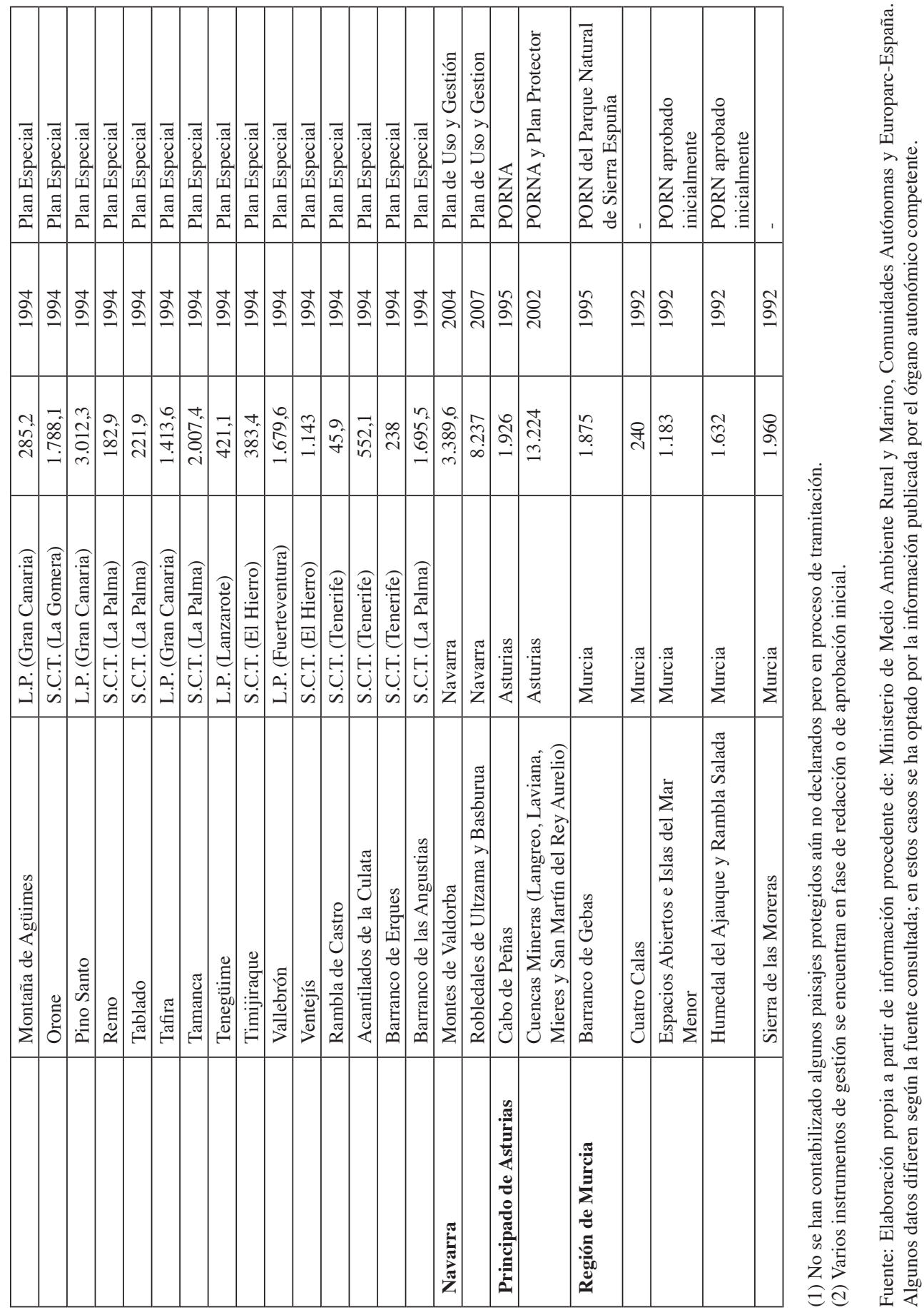


natural y cultural: las Montañas del Maigmó y del Sit, la Sierra de Bernia y Ferrer, o el río Serpis, son buenos ejemplos de paisajes excepcionales cuyos usos y actividades humanas -cada vez más intensas- requieren de una regulación que garantice la preservación de los valores que los singularizan.

Por último, comparten argumentos similares las actuaciones llevadas a cabo en Aragón (Pinares de Rodeno; San Juan de la Peña y Monte Oroel), Galicia (Penedos de Pasarela e Traba), Navarra (Montes de Valdorba; Robledales de Ultzama y Basaburua) y Asturias (Cuencas Mineras), si bien es cierto que en alguna de estas regiones también se han impulsado declaraciones que responden esencialmente a razones de conservación de elementos naturales singulares, casos del Cabo de Peñas (Asturias) o de las Foces de Fago y Biniés (Aragón).

b) En segundo término, en algunas autonomías se ha entendido que el Paisaje Protegido ha de ser una figura orientada prioritariamente a la salvaguarda de valores destacados del medio natural, en la línea de los últimos casos citados. Con carácter general, éste ha sido el criterio seguido en Extremadura (Monte Valcorchero), Castilla-La Mancha (Chorrera de Horcajo) y Murcia. En esta última región la preservación de elementos bióticos y geomorfológicos ha sido, claramente, el leitmotiv de la declaración de sus cinco Paisajes Protegidos (los antes citados más el Barranco de Gebas) (Espejo y García, 2011). Quizás por ello en Murcia se ha fijado el PORN como instrumento para el manejo y gestión de los mismos, supuesto que se adapta mejor a las categorías protectoras que priman la conservación sobre otros fines (no se olvide que en la legislación estatal sólo es considerado obligatorio para Parques y Reservas Naturales).

c) Finalmente, una tercera opción se ha concretado en la declaración de tramos fluviales singulares como Paisajes Protegidos, primando su funcionalidad como corredores ecológicos entre ámbitos distantes que gozan de una alta valoración ambiental. Esta estrategia evidencia una clara influencia de la Directiva de Hábitats, donde se aboga reiteradamente por dotar de coherencia y conectividad a los espacios que integran la Red Natura europea. Así, los Paisajes Protegidos andaluces del Corredor Verde del Guadiamar (Feria, 2004) y del Río Tinto responden claramente a esta concepción; han sido delimitados como arterias de conexión entre espacios naturales valiosos, para facilitar el desplazamiento de la fauna entre tales ámbitos, y, a la vez, garantizar la regeneración ambiental de unos ecosistemas fluviales que se encuentran muy degradados a causa de una antigua e intensa actividad minera. También el primer Paisaje Protegido gallego (Valle del Navea) se encuadra en estos planteamientos, ya que su declaración vino dada por su función como corredor fluvial de enlace entre el macizo central orensano y el sistema fluvial Miño-Sil, área importante de localización de diferentes tipos de hábitats de interés comunitario.

Por añadidura, las diferencias conceptuales que planean sobre esta figura se manifiestan también en la variedad de los instrumentos de gestión establecidos por las Comunidades Autónomas. En la actualidad, la mayoría de paisajes protegidos se rige conforme a algún tipo de plan, entre los que destaca el Plan Especial aplicado en Canarias, definido como el «instrumento de planeamiento de los paisajes protegidos, que incluye los usos del territorio en toda su extensión (...); en el mismo pueden establecerse zonas diferenciadas según sus exigencias de protección definiendo regímenes de usos distintos de acuerdo a la zonificación 
Figura 1

\section{PAISAJES PROTEGIDOS DECLARADOS EN ESPAÑA (2010)}

ANDALUCÍA

1. Corredor Verde del Guadiamar

2. Río Tinto

ARAGÓN

3. Pinares de Rodeno

4. San Juan de la Peña y Monte Oroel

5. Foces de Fago y Biniés

CASTILLA-LA MANCHA

6. Chorrera de Orcajo

COMUNIDAD DE VALENCIA

7. Río Serpis

8. Sierra del Maigmó y Sierra del Sit

9. Puigcampana y el Ponotx

10. Sierra de Bernia y Ferrer

11. Les Sorts

12. Solana del Benicadell

13. Umbría del Benicadell

14. Desembocadura del Millars

EXTREMADURA

15. Monte Valcorchero

GALICIA

16. Valle del Río Navea

17. Penedos de Pasarela e Traba

\section{NAVARRA}

45. Montes de Valdorba

46. Robledales de Ultzama y Basburua

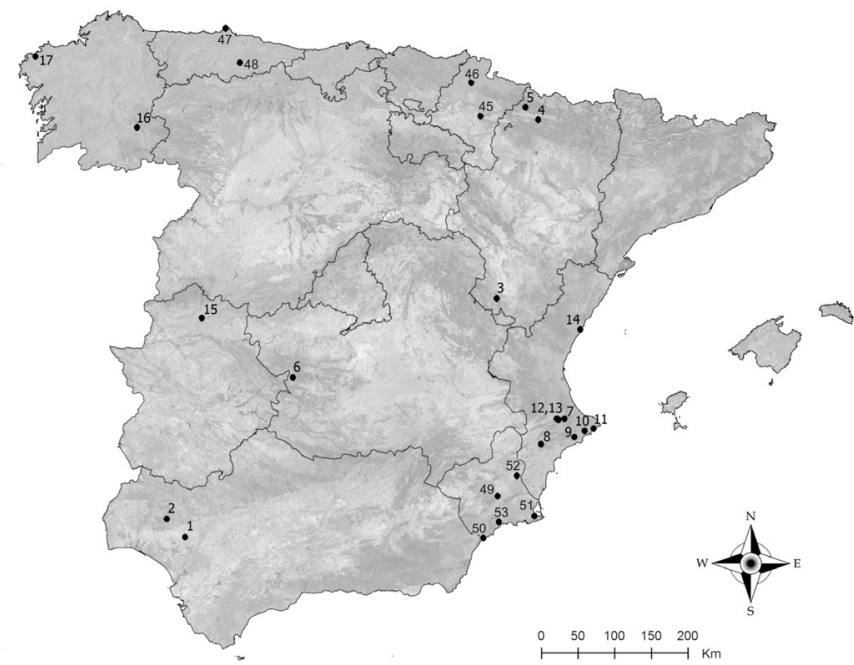

PRINCIPADO DE ASTURIAS

47. Cabo de Peñas

48. Cuencas Mineras (Langreo, Laviana, Mieres y San Martín del Rey Aurelio)

REGIÓN DE MURCIA

49. Barranco de Gebas

50. Cuatro Calas

ISLAS CANARIAS

51. Espacios Abiertos e Islas del Mar Menor

52. Humedal del Ajauque y Rambla Salada

53. Sierra de las Moreras

18. Fataga

19. La Geria

20. La Isleta

21. Las Cumbres

22. Lomo Magullo

23. Malpaís Grande

24. Montaña de Agüimes

25. Pino Santo

26. Tafira

27. Tenegüime

28. Vallebrón

29. Acantilados de la Culata

30. Barranco de Erques

31. Barranco de las Angustias

32. Costa de Acentejo

33. Ifonche

34. La Resbala

35. Las Lagunetas

36. Las Siete Lomas

37. Los Capeches, Tigaiga y Ruiz

38. Orone

39. Rambla de Castro

40. Remo

41. Tablado

42. Tamanca

43. Timijiraque

44. Ventejís

Fuente: Elaboración propia. 
establecida en la normativa vigente». En definitiva, los Planes Especiales aspiran a ordenar los usos del suelo - con la intención de garantizar la estabilidad paisajística- antes que proteger componentes concretos del medio natural. En Valencia, Aragón y Navarra se ha optado, desde un planteamiento bastante similar al canario, por el Plan Rector de Uso y Gestión, cuya función principal es zonificar el espacio protegido y establecer un régimen de usos para cada sector; éste ha sido también el instrumento empleado mayoritariamente en Parques, Reservas Naturales, y otras figuras protectoras de corte autonómico. Por otro lado, en algunas regiones el referente es un Plan de Ordenación de los Recursos Naturales (Murcia, Asturias), una elección poco apropiada en nuestra opinión, considerando que en la designación de los Paisajes Protegidos los componentes culturales y estéticos del territorio han de tener un peso esencial. Sin embargo, es preciso aclarar la peculiaridad de Asturias al respecto, una región donde la totalidad de los espacios protegidos tiene como documento marco para su manejo el Plan de Ordenación de los Recursos Naturales de Asturias (PORNA), sin que ello impida la promulgación de instrumentos específicos para aquellos casos que así lo requieran; valga como ejemplo el Plan Protector aprobado en 2004 para el Paisaje Protegido de las Cuencas Mineras. Finalmente, también se dan situaciones (Andalucía, Castilla-La Mancha, Extremadura, Galicia) donde el manejo de los Paisajes Protegidos se está llevando a cabo sin ningún tipo de referente planificador, aunque en algún caso (Planes de Conservación estipulados en la normativa gallega) esté previsto y en fase de elaboración.

\section{CONCLUSIONES}

En el marco de las políticas españolas de protección de espacios naturales, la preocupación por preservar determinados paisajes considerados especialmente representativos -en orden a su carácter identitario, la singularidad de sus valores naturales y/o culturales, sus componente estéticos, etc.- ha estado presente desde el mismo momento de la gestación de aquéllas, si bien es cierto que normalmente se ha tratado de manifestaciones aisladas, bajo formulaciones poco desarrolladas y sin un claro respaldo normativo. Sin embargo, desde la década de los ochenta del pasado siglo, se produce un considerable avance en el reconocimiento de los valores vinculados al paisaje; progresivamente ha ido calando en la sociedad española un mejor entendimiento de este concepto, en el que ya se reconoce un elemento patrimonial de primer orden.

Como reflejo de lo anterior, desde los ámbitos político y científico se viene insistiendo en la necesidad de inventariar, proteger y gestionar el patrimonio paisajístico y pese a que a escala estatal aún no existe una legislación expresa que contemple al paisaje como un bien jurídico (Zoido, 2010), lo cierto es que se ha reforzado considerablemente su regulación, bien a través de aportaciones parciales recogidas en la legislación estatal de carácter sectorial, bien mediante la promulgación de leyes específicas por parte de algunas Comunidades Autónomas. En este contexto, el análisis efectuado en las páginas precedentes viene a destacar que, a nivel teórico y programático, la preservación del paisaje ha alcanzado el rango de principio inspirador de las leyes marco de conservación del medio natural promulgadas desde finales de los ochenta; un logro estructural que se ha visto acompañado de medidas concretas de considerable calado, entre las que sobresalen: la creación de una categoría protectora específica -el Paisaje Protegido-, la obligatoriedad de incluir en los Planes de 
Ordenación de los Recursos Naturales un inventario/diagnóstico de los paisajes representativos existentes en sus ámbitos de influencia, o el reconocimiento de medidas incentivadoras para todas aquellas actividades que redunden en la protección del paisaje, y que se realicen tanto en el interior de los espacios naturales protegidos como de los ámbitos en los que se implanten acuerdos de custodia del territorio.

Sin embargo, no es menos cierto que pese a los logros alcanzados en el ámbito de la legislación básica, el desarrollo de las actuaciones concretas en la materia ha sido insuficiente y ha estado presidido por cierta confusión. La raíz de ello se encuentra, a nuestro juicio, en la incertidumbre generada por una laxa definición de la figura de Paisaje Protegido, y también por una escasa reflexión y debate en torno al significado que debe tener el paisaje en las políticas de espacios naturales protegidos.

Así, entre las carencias principales de orden normativo y planificador, es llamativa la escasa atención prestada al paisaje en las diferentes leyes autonómicas de espacios protegidos que se han ido promulgando desde los años ochenta, ignorándose de esta forma el camino abierto por la legislación estatal, con alguna loable excepción como la Canaria. En el mismo sentido, es sabido que con carácter general ni los PORN ni los PRUG -instrumentos básicos reguladores de la inmensa mayoría del territorio protegido en España- se han ocupado de inventariar, diagnosticar y regular el patrimonio paisajístico presente en sus respectivos ámbitos de influencia. Y ni que decir tiene, por otra parte, que la coordinación en materia de paisaje entre estos y los restantes instrumentos autonómicos de ordenación territorial aprobados en las dos últimas décadas, ha brillado por su ausencia; un ejemplo antológico de ello lo encontramos en Andalucía, con una evidente falta de directrices comunes entre PORN y PRUG -planificación medioambiental- y Planes Subregionales de Ordenación del Territorio -planificación territorial-.

En lo referido a las actuaciones protectoras concretas, lo cierto es que la figura de Paisaje Protegido ha tenido un escaso desarrollo en España, transcurridas más de dos décadas desde su creación. De una parte, el número de declaraciones ha aumentado de forma progresiva, pero realmente sin las aportaciones de Canarias y, en menor medida, Valencia, el catálogo español de paisajes protegidos sería muy pobre. En la actualidad es la categoría protectora de ámbito estatal menos empleada -con la excepción de las Áreas Marinas Protegidas, de creación reciente- a pesar de sus indudables atractivos y potencialidades. No se olvide que siete Comunidades Autónomas aún no han hecho uso de la misma, y que las restantes la han aplicado conforme a criterios muy heterogéneos, patentes tanto en la selección como en la gestión de los espacios designados. Así, en Andalucía -la región con más superficie protegida: 150 espacios naturales con aproximadamente 1,7 millones de ha- sólo se han declarado dos Paisajes Protegidos, y ello conforme a una interpretación propia y singular de lo que debe ser esta figura protectora.

A nuestro juicio, la cuestión esencial que debe plantearse es si resulta coherente la existencia de una figura como la de Paisaje Protegido y, en caso afirmativo, si ésta debe regularse a través de la legislación específica de conservación del medio natural -actual Ley del Patrimonio Natural y de la Biodiversidad-; téngase en cuenta que, entre otras, las directrices emanadas del Convenio Europeo del Paisaje establecen criterios variados y complejos para la definición y selección de los paisajes representativos, que sobrepasan claramente las competencias y los objetivos de los organismos medioambientales. 


\section{BIBLIOGRAFÍA}

ACOSTA BONO, G. (2007): «El paisaje en los instrumentos de planificación sectorial y de ordenación del territorio». Actas del I Congreso Paisaje e Infraestructuras. Sevilla. Consejería de Obras Públicas y Transporte de la Junta de Andalucía, pp. 131-165

ARIAS IBÁÑEZ, E. (2007): «La planificación en los espacios naturales protegidos: aplicación de los PORN en las Cordilleras Béticas andaluzas». Investigaciones Geográficas, $\mathrm{n}^{\circ}$ 44, pp. 103-127.

ARIAS, E. y GARZÓN, R. (2009): Planificación y cambios territoriales y paisajísticos en un área de singular protección: La Sierra de las Nieves (Málaga). En: Geografía, Territorio y Paisaje: el estado de la cuestión. XXI Congreso de Geógrafos Españoles. Ciudad Real, Universidad de Castilla-La Mancha y Asociación de Geógrafos Españoles, pp. 51-67.

BERMEJO LATRE, J.L. (2007): «Legislación del paisaje en España: retos y perspectivas». Actas del I Congreso Paisaje e Infraestructuras. Sevilla. Consejería de Obras Públicas y Transporte de la Junta de Andalucía, pp. 429-448.

CABALAR FUENTES, M. (2007): «La figura de paisaje protegido en Galicia. Criterios empleados en su aplicación». En: Geografía, Territorio y Paisaje: el estado de la cuestión. XXI Congreso de Geógrafos Españoles. Ciudad Real, Universidad de Castilla-La Mancha y Asociación de Geógrafos Españoles, pp. 831-843.

CASADO DE OTAOLA, S. (1997): Los primeros pasos de la ecología en España. Madrid, MAPA (Serie Estudios no 128), 529 pp.

CONSEJO DE EUROPA (2000): Convención Europea del Paisaje. Florencia, Consejo de Europa.

ESPEJO MARÍN, C. y GARCÍA MARÍN, R. (2011): «Intentos de gobernanza de un espacio mediterráneo sometido a gran presión: el caso del Mar Menor en Murcia». En: FARINÓS, J. (Ed. y Coord.) La Gestión Integral de Zonas Costeras ¿Algo más que una ordenación del litoral revisada?, Valencia, Universitat de Valencia, pp. 291-319.

FERIA TORIBIO, J.M. (2004): «El corredor verde del Guadiamar y las relaciones entre protección y ordenación del territorio». Estudios Geográficos, nº 256, pp. 445-469.

FROLOVA, M., MENOR TORIBIO, J. y CANCER POMAR, L. (2003): «El paisaje en las políticas públicas de Francia y España: desde la protección del monumento a la gestión del espacio». Estudios Geográficos, n 253,pp. 605-622.

GARCÍA ÁLVAREZ, J. (2009): Los valores sintéticos del paisaje en la caracterización de los primeros espacios naturales protegidos de España. El caso del Parque Nacional de la montaña de Covadonga. En: Geografía, Territorio y Paisaje: el estado de la cuestión. XXI Congreso de Geógrafos Españoles. Ciudad Real, Universidad de Castilla-La Mancha y Asociación de Geógrafos Españoles, pp. 1.037-1.054.

GÓMEZ MENDOZA, J. (1999): «Paisajes y espacios protegidos en España». Boletín de la Institución Libre de Enseñanza, $\mathrm{n}^{\circ}$ 34-35, pp. 131-152.

LASAGABASTER, I. y LAZCANO, I. (2004): «Protección del paisaje y espacios naturales protegidos. Revista Vasca de Administración Pública, n ${ }^{\circ}$ 70, pp. 125-187.

MARTÍNEZ DE PISÓN, E. y ORTEGA, N. (Eds.) (2007): La conservación del paisaje en los Parques Nacionales. Madrid, Universidad Autónoma de Madrid-Fundación Duques de Soria, $237 \mathrm{pp}$. 
MATA OLMO, R. (2000): «Los orígenes de la conservación de la naturaleza en España. En: Martínez de Pisón, E. y Sanz, C. (Eds.) (2000): Estudios sobre el paisaje. Madrid, Universidad Autónoma de Madrid-Fundación Duques de Soria, pp. 259-280.

MULERO MENDIGORRI, A. (2002): La protección de espacios naturales en España. Madrid, Mundi-Prensa, 309 pp.

MULERO MENDIGORRI, A. (2009): Espacios naturales protegidos y ordenación del litoral en Andalucía. Córdoba, Servicio de Publicaciones Universidad de Córdoba, 249 pp.

ORTEGA, N. y GARCÍA, J. (2009): «Paisajes y lugares de memoria: Covadonga y El Paular». En: Martínez de Pisón, E. y Ortega, N. (Eds.) Los valores del paisaje. Madrid, Universidad Autónoma de Madrid-Fundación Duques de Soria, pp. 45-93.

RAMIS, C.I. et al. (2009): «Análisis y evaluación de los instrumentos legislativos de protección, gestión y ordenación del paisaje». En: Geografía, Territorio y Paisaje: el estado de la cuestión. XXI Congreso de Geógrafos Españoles. Ciudad Real, Universidad de Castilla-La Mancha y Asociación de Geógrafos Españoles, pp. 1.323-1336.

RUBIO, P. y MUÑOZ, J. (2008): «Gestión del paisaje en áreas de interés natural». Cuadernos Geográficos, n 43 , pp. 271-288.

SOLÉ, J. y BRETÓN SOLO DE ZALDIVAR, V. (1986): «El paraíso poseído. La política española de parques naturales (1880-1935). Geo-crítica, nº 63, 59 pp.

TROITIÑO, M.A. et al. (2005): «Los espacios protegidos en España: Significación e incidencia territorial». Boletín de la Asociación de Geógrafos Españoles, n 39, pp. 227-265.

ZOIDO NARANJO, F. (2001): «La Convención Europea del Paisaje y su aplicación en España». Ciudad y Territorio. Estudios Territoriales, n ${ }^{\circ}$ 128, pp. 275-281.

ZOIDO NARANJO, F. (2010): «Territorio y paisaje, conocimiento, estrategias y políticas». En: Territorio, paisaje y sostenibilidad.XXI Congreso Nacional de Geografía-Ponencias. Barcelona, Ediciones del Serbal, pp. 85-112.

ZOIDO, F. y VENEGAS, C. (Coord.) (2002): Paisaje y Ordenación del Territorio. Sevilla, COPT (Junta de Andalucía)-Fundación Duques de Soria. 
